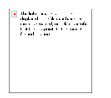

\title{
Multi-objective optimization of the operation and maintenance assets of an offshore wind farm using genetic algorithms
}

\begin{tabular}{|r|l|}
\hline Journal: & Wind Engineering \\
\hline Manuscript ID & WIE-18-0041 \\
\hline Manuscript Type: & Research Article \\
\hline Date Submitted by the Author: & 01-Jun-2018 \\
\hline Complete List of Authors: & $\begin{array}{l}\text { Rinaldi, Giovanni; University of txeter College of tngineering Mathematics } \\
\text { and Physical Sciences, } \\
\text { Pillai, Ajit } \\
\text { Thies, Philipp } \\
\text { Johanning, Lars }\end{array}$ \\
\hline Keywords: & $\begin{array}{l}\text { Operation and maintenance, Assets management, Offshore wind, } \\
\text { Optimization, Genetic algorithms, Decision-making }\end{array}$ \\
\hline Abstract: & $\begin{array}{l}\text { This paper explores the use of genetic algorithms to optimize the operation } \\
\text { and maintenance (O\&M) assets of an offshore wind farm. Three different } \\
\text { methods are implemented in order to demonstrate the approach. The } \\
\text { optimization problem simultaneously considers both the reliability } \\
\text { characteristics of the offshore wind turbines and the composition of the } \\
\text { maintenance fleet, seeking to identify the optimal configurations for the } \\
\text { strategic assets. } \\
\text { These are evaluated in order to minimize the operating costs of the } \\
\text { offshore farm while maximizing both its reliability and availability. } \\
\text { The considerations used for the application of genetic algorithms as an } \\
\text { effective way to support the assets management are described, and a case } \\
\text { study to show the applicability of the approach is presented. } \\
\text { The variation of the economic performance indicators as a consequence of } \\
\text { the optimization procedure are discussed, and the implementation of this } \\
\text { method in a wider computational framework for the O\&M assets } \\
\text { improvement introduced. }\end{array}$ \\
\hline \hline
\end{tabular}




\title{
Multi-objective optimization of the operation and maintenance assets of an offshore wind farm using genetic algorithms
}

\author{
Giovanni Rinaldi ${ }^{1}$, Ajit C. Pillai ${ }^{1}$, Philipp R. Thies ${ }^{1}$ and Lars Johanning ${ }^{1}$
}

\begin{abstract}
This paper explores the use of genetic algorithms to optimize the operation and maintenance (O\&M) assets of an offshore wind farm. Three different methods are implemented in order to demonstrate the approach. The optimization problem simultaneously considers both the reliability characteristics of the offshore wind turbines and the composition of the maintenance fleet, seeking to identify the optimal configurations for the strategic assets. These are evaluated in order to minimize the operating costs of the offshore farm while maximizing both its reliability and availability. The considerations used for the application of genetic algorithms as an effective way to support the assets management are described, and a case study to show the applicability of the approach is presented. The variation of the economic performance indicators as a consequence of the optimization procedure are discussed, and the implementation of this method in a wider computational framework for the O\&M assets improvement introduced.
\end{abstract}

\section{Keywords}

Operation and maintenance, offshore wind, genetic algorithm, optimization, decision-making

\section{Introduction}

As offshore wind continues its path of cost reduction, while global energy demand and the need for carbon neutral power plants increase, the installation of further offshore wind projects (OWPs) is anticipated in the coming years. The trends clearly indicate that offshore wind farms (OWFs) occupying a larger area, with wind turbines of higher rated power, located in deeper waters, and at larger distances to shore, will be favoured (1). Due to their significant contribution to the operational expenditures (OpEx) and to the overall cost of energy, operation and maintenance $(\mathrm{O} \& \mathrm{M})$ of the devices will play a pivotal role in determining the profitability of a project (2). For this reason, flexible and comprehensive tools are needed to grasp the complex dynamics of the OWF and provide support in the choice and dispatch of the O\&M assets.

With regard to such choices, several options exist for wind turbine developers concerned with the design of the device and its features. These aim towards the maximization of the reliability, in order to achieve high values of availability, while keeping the price per turbine low. On a different note, various alternatives exist for OWF owners or operators committed to the selection of access and maintenance systems (e.g. vessels and helicopters) needed to keep the devices functional and efficient for the desired lifetime. As a consequence, computer aided models which aim to characterize these parameters and to support the decision making process have been of increasing interest in recent years. Some of these tools are problem-specific in order to address one of the aspects needed to define the final O\&M strategy, e.g. vessels logistics $(3 ; 4)$, spare parts allocation (5) or planned interventions $(6 ; 7)$. Others exploit different solution techniques to cover several aspects of the maintenance and assets management at the same time $(8 ; 9 ; 10 ; 11)$. Comprehensive reviews and classification frameworks of these models can be found in Hofman, 2011 (12) and Shafiee and Sørensen, 2017 (13). With the intention of reducing the uncertainties in existing models and provide a generalized method applicable to all offshore renewable energy technologies (wind, wave and tidal energy devices), the authors proposed a computational model for the characterization of the O\&M assets of an offshore renewable energy farm, as described in $(14 ; 15)$ and henceforth referred to as characterization tool. This tool allows for the estimation of the key performance indicators (KPIs) of the farm through implementation of a probabilistic assessment based on the Monte Carlo technique (16) and taking into account the reliability data of the devices and the capabilities of the maintenance assets.

However, even when all the characteristics of an OWF are depicted and possible areas for improvements identified, the proposal of alternative solutions to the current maintenance strategy and assets selection relies on the judgement of decision-makers. These, according to their expertise and experience, have to interpret the results obtained with the characterization tool, propose an alternative choice in the assets of the farm which should improve the maintainability

\footnotetext{
${ }^{1}$ Renewable Energy Group, College of Engineering, Mathematics and Physical Science, University of Exeter, Cornwall Campus, Penryn TR10
} 9EZ, UK

\section{Corresponding author:}

Giovanni Rinaldi

Email: G.Rinaldi@exeter.ac.uk 
or profitability of the OWP, and test the new configuration to assess the extent of the potential improvement. Therefore, in order to automate this process and reduce the possibility of leaving possible improved solutions unexplored, the authors propose the use of multi-objective optimization by means of genetic algorithms (GAs) (17). In fact, to the best of the authors knowledge, though these techniques have been applied to a number of offshore renewables related optimization problems $(18 ; 19 ; 20 ; 21)$, no previous application for the improvement of the assets management, aimed at the improvement of the O\&M strategies, is known. Specific objective functions for the evaluation of the effectiveness of an O\&M strategy are therefore implemented. As the optimal values of the decision variables which describe the OWF assets is uncertain, the end goal of this optimization process is to attain the optimal values of these decision variables. Consequently, the aim of this paper is twofold. First, to demonstrate the applicability of GAs as an efficient way to facilitate the selection of OWF assets by showing the results obtained with a case study. Secondly, the presented methodology seeks to provide a viable alternative to the use of computationally expensive O\&M characterization tools through the implementation of a simpler and faster surrogate model based on evaluation functions. It must be noticed that the assets management decision problem, intended as choice of the ideal value for each of the decision variables, is investigated, as opposed to the scheduling of the maintenance operations taking into account corrective, preventive and predictive maintenance.

Section 2 introduces the methodology deployed for this work, together with the comparison of three GA architectures and the description of the objective functions implementation. Subsequently, the results which are obtainable using this methodology are presented in section 3 through a case study representing the two phases of an existing and projected OWF. The results are then discussed and conclusions on the use of this technique drawn in sections 4 and 5 .

\section{Methodology}

Genetic algorithms were initially developed by John Holland in the early 1970's (22). These are adaptive search procedures which mimic biological processes of selection and evolution to solve both constrained and unconstrained optimization problems. Based on an analogous operating principles, the GAs consider a population of solutions which through a series of steps evolve over time to reach the optimal solution. In a manner similar to how biological species adapt to their environment and preserve beneficial traits between subsequent generations, a GA uses the information of how solutions perform in order to guide the search through the search space. Thus, they can be used in complex singleobjective and multi-objective problems in order to generate solutions that have evolved towards the optimal result (23). A typical GA works according to the flowchart shown in Figure 1. Firstly, a population, a group of individuals, is created at random. In this, each individual represents a candidate solution to the decision problem and it is encoded using binary code or other representations (24). Secondly, each individual is evaluated according to its suitability with respect to a predefined objective, and a score (fitness) is assigned to it. Individuals are assigned a probability of selection based on their fitness scores. Generally speaking, individuals with higher fitness scores have higher probabilities of selection, but various selection principles exist. After selection, pairs of individuals are recombined through crossover algorithms to generate the new individuals of the population. In this work, a single point crossover is used; in this a "crossover point" is randomly selected, and bits from the beginning of the chromosome to the crossover point are copied from one parent while the remaining bits are copied from the second parent. Finally, the new individuals are randomly mutated to refine local searches in the investigated domain. Mutation is achieved through the inversion of a certain number of bits selected at random, proportional to the mutation rate.

This process is repeated until specified termination conditions are met or the maximum number of generations is reached. In this way, over successive generations, the population evolves towards a set of improved solutions. Optionally, a restricted number of individuals with the best fitness values, called elite, can be preserved from one generation to another without being subjected to the genetic operators.

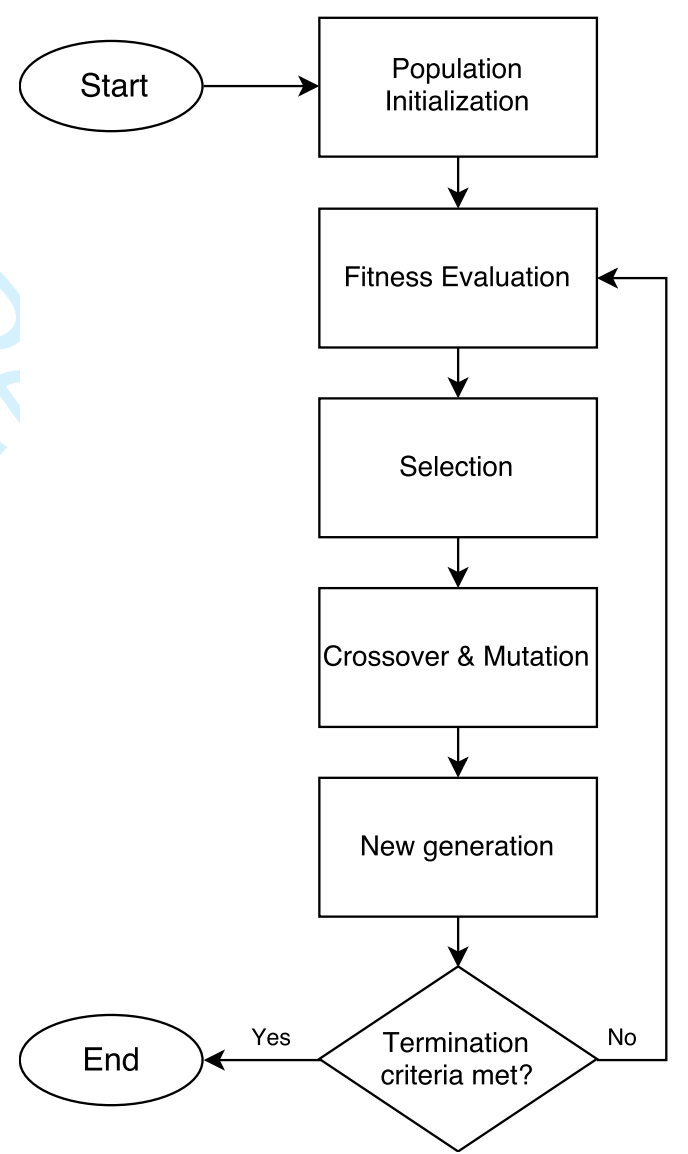

Figure 1. Flowchart of a genetic algorithm.

In order to take into account the requirements and technical implications in selecting the most appropriate combination of assets for an OWF, multiple criteria must be considered. In fact, in order to increase the longevity and profitability of a project, the direct O\&M costs deriving from the choice of the maintenance systems and possible interventions on the devices have to be kept as 
low as possible, while specific targets of reliability are met and the availability of the devices is as high as possible in order to reduce the lost production due to downtime. As a result, the problem becomes a multiobjective optimization aimed at minimizing the costs while maximizing both the reliability and the availability. Several multi-objective strategies using GAs exist (25), and to explore their suitability for offshore O\&M problems, three are implemented and compared in this work. These are here referred to as: 1)Superposition method, 2)Weighted sum method and 3)VEGA inspired method. The first one, already introduced in (17), superposes the results obtained by executing successive single-objective optimizations for each of the individual objectives considered. In this work, the individual objectives considered are:

- Minimization of costs;

- Maximization of reliability;

- Maximization of availability;

- Minimization of costs / reliability ratio; and

- Minimization of costs / availability ratio.

When considering the superposition approach, the GA framework illustrated in Figure 1 is applied separately for each one of these objectives. Exclusively for the superposition approach, two of the objectives represent the ratio between other objective functions. This is done in order to orientate the search in different areas of the investigated objective space, as exemplified in Figure 2. The results found with each single-objective optimization are then combined, and a set of solutions approximating the Pareto frontier is selected. In theory, the true Pareto frontier represents the optimal trade offs between the competing objectives. It must be noticed that generally, using GAs, it cannot be guaranteed that the Pareto front found at the end of the optimization procedure corresponds with the optimal solutions for the investigated problem, but only that the set of best solutions are non-dominated (cannot be further improved with respect to one of the objectives without worsening at least with respect to another) with respect to all the solutions identified during the search. For some specific problems, other techniques, e.g. linear programming, can be used to find the real Pareto optimal solutions and measure the distance from the non-dominated solutions found with the GA. However, for sake of simplicity, in this work the non-dominated front found with the GA will be referred to as the Pareto front.

This process is illustrated in the flowchart in Figure 3. The solutions lying on the Pareto frontier are considered as optimal in the wider sense that no other solutions in the search space are superior to them when all objectives are considered simultaneously (26). In other words, a solution belongs to the Pareto frontier if there is no way to further improve one objective without worsening at least one other.

The second approach considered in this work is the weighted sum method (27). This consists in a linearization of the problem by assigning a 'weight' $\omega_{i} \in[0,1]$ to each objective function, such that $\Sigma_{i} \omega_{i}=1$ and the fitness of each solution is calculated by summing up the scores with respect to all the weighted objective values $\omega_{i} \cdot f_{i}(x)$. The principal challenge of using this approach is the selection of the appropriate weights for each objective function, since
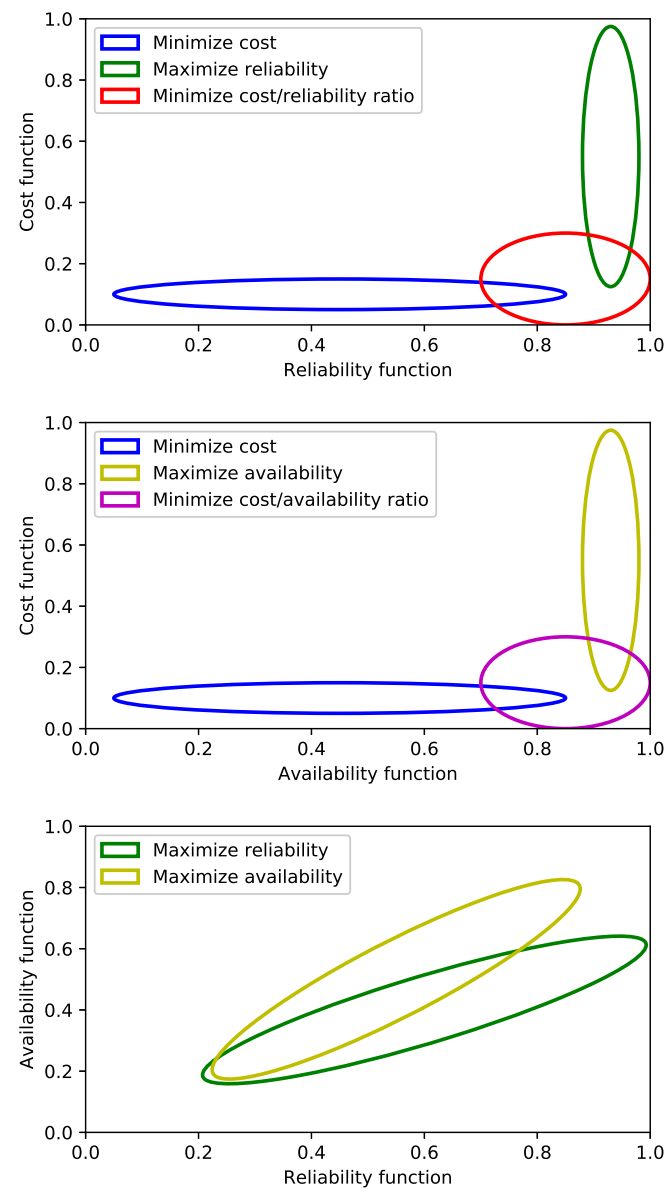

Figure 2. Example of areas of the objective space where the search for new solutions focuses depending on the objective function.

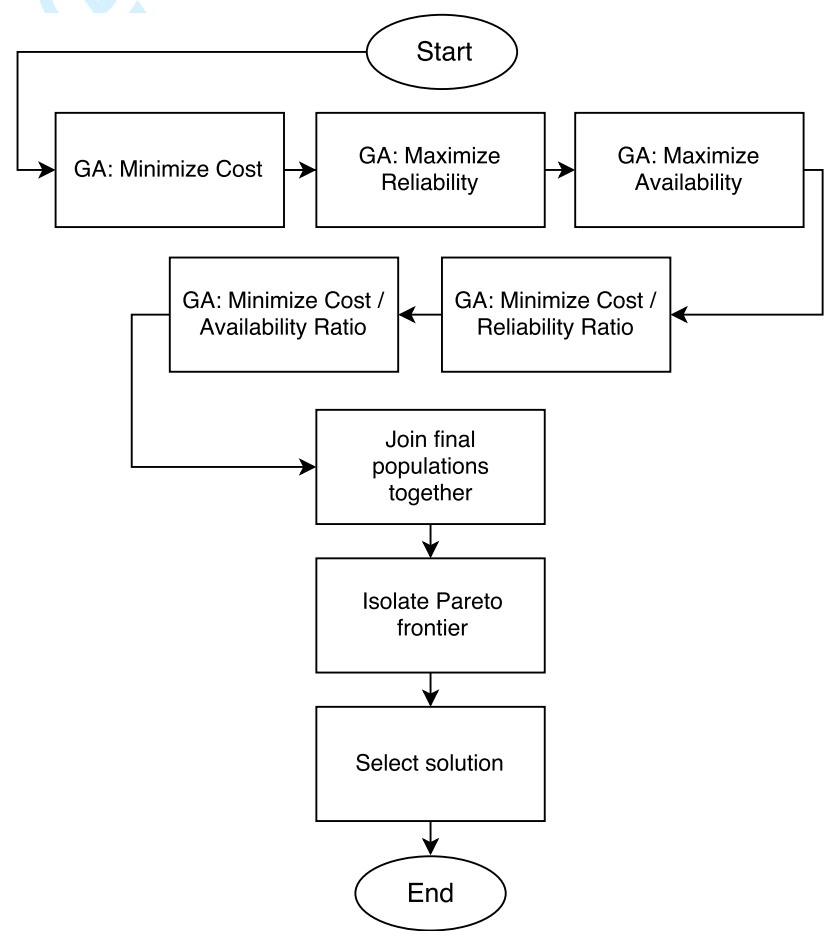

Figure 3. Flowchart of the approach exploiting individual single-objectives optimizations. 
the value of each weight corresponds to the priority given to an objective. As a result, similarly to what is shown in Figure 2, each set of weights will orient the search towards a different region of the search space. Thus, in order to overcome this difficulty and to not favour explicitly one of the above mentioned criteria (cost, reliability, availability) over the others, an iterative process in which the weights of the different functions are changed randomly at each iteration is implemented. All the results obtained at each iteration are then combined in order to obtain the complete picture for the explored search space during all the iterations and the Pareto solutions selected. This process is depicted in the flowchart in Figure 4. Another drawback of this approach is that, once the problem is linearized by including all the objective functions and their weights in a single vector, only one objective can be considered, i.e. either maximization or minimization of the resulting vector. Therefore the problem has to be rewritten in the following way:

$$
\begin{aligned}
& \text { Maximize: } J(x)=w_{c} \cdot \frac{1}{f_{c}(x)}+w_{r} \cdot f_{r}(x)+w_{a} \cdot f_{a}(x) \\
& \text { Subject to: } w_{c}, w_{r}, w_{a}>=0 ; w_{c}+w_{r}+w_{a}=1 \\
& \text { Where: } f_{c}=\text { cost function } \\
& f_{r}=\text { reliability function } \\
& f_{a}=\text { availability function }
\end{aligned}
$$

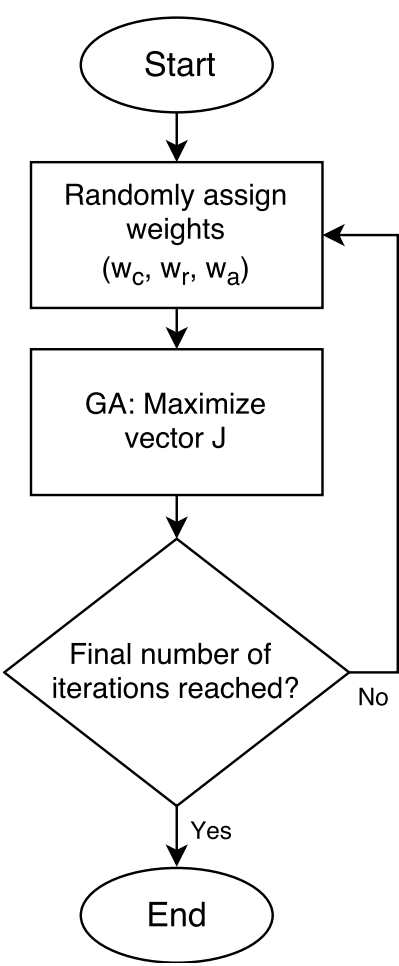

Figure 4. Flowchart of the approach exploiting the weighted sum method.

and the values of the objective functions are normalized with respect to the maximum value obtainable for each objective (previously calculated according to the input parameters) in order to consider them equitably and avoid potential domination by one another.
Finally, the third approach considered is inspired to the Vector Evaluated Genetic Algorithm (VEGA) (28), and its process is illustrated in the flowcharts in Figures 5 and 6. In this approach, a different score is assigned for each one of the objective functions considered to each individual of the population. The initial population is then split into $k$ sub-populations (where $k$ is the number of objectives) by picking the first $n / k$ individuals (where $n$ is the size of the population) sorted according to the results of each evaluation. A new population is then obtained by recombining these sub-populations and the GA can continue as usual, with crossover and mutation of selected individuals. Similar to the previous approaches, the objective functions considered in this work are the minimization of the cost, the maximization of the reliability and the maximization of the availability. The selection of the individuals for mating (crossover) is carried out through two options. In the first variant, the two individuals ("parents") are randomly selected by picking one each from two of the three sub-populations obtained before these are shuffled together, whereas in the second variation a selection process based on the roulette wheel method (29) is used after the three sub-populations are shuffled together. Analogously to the previous approaches, the results of both variations are combined together to take advantage of the different areas of the search space explored. In this way, the extents of the search space are investigated, a quality desirable at the beginning of the search procedure before the search converges towards improved solutions, and the exclusive selection of individuals that excel in only one of the three objectives is avoided. As a consequence, also the selection of individuals which are moderately good with respect to all the objective functions, and thus may be useful to find compromise solutions, is ensured.

For all the three approaches, the parameters to control the execution of the GA are assigned according to guidance given in the literature (30) and then further tuned in order to achieve a good balance between extensiveness of the search and computational time for this specific problem implementation. A series of preliminary tests based on a sensitivity analysis of the best and average fitness values has been used for this purpose, taking into account their absolute value and, since the two values should ideally converge, the relative difference between them. These control parameters are given in Table 1.

Table 1. GA control parameters.

\begin{tabular}{cc}
\hline Parameter & Value \\
\hline Generations & 30 \\
Population size & 50 \\
Elite individuals & 5 \\
Crossover rate & 0.84 \\
Mutation rate & 0.01 \\
Encoding & Binary \\
\hline
\end{tabular}

\section{Objective functions}

Three objective functions are considered in this work in order to adequately represent the problem of improving the assets selection and logistics management of an OWF. To evaluate the fitness of each individual (i.e. potential solution), an accurate implementation of the functions relating the 


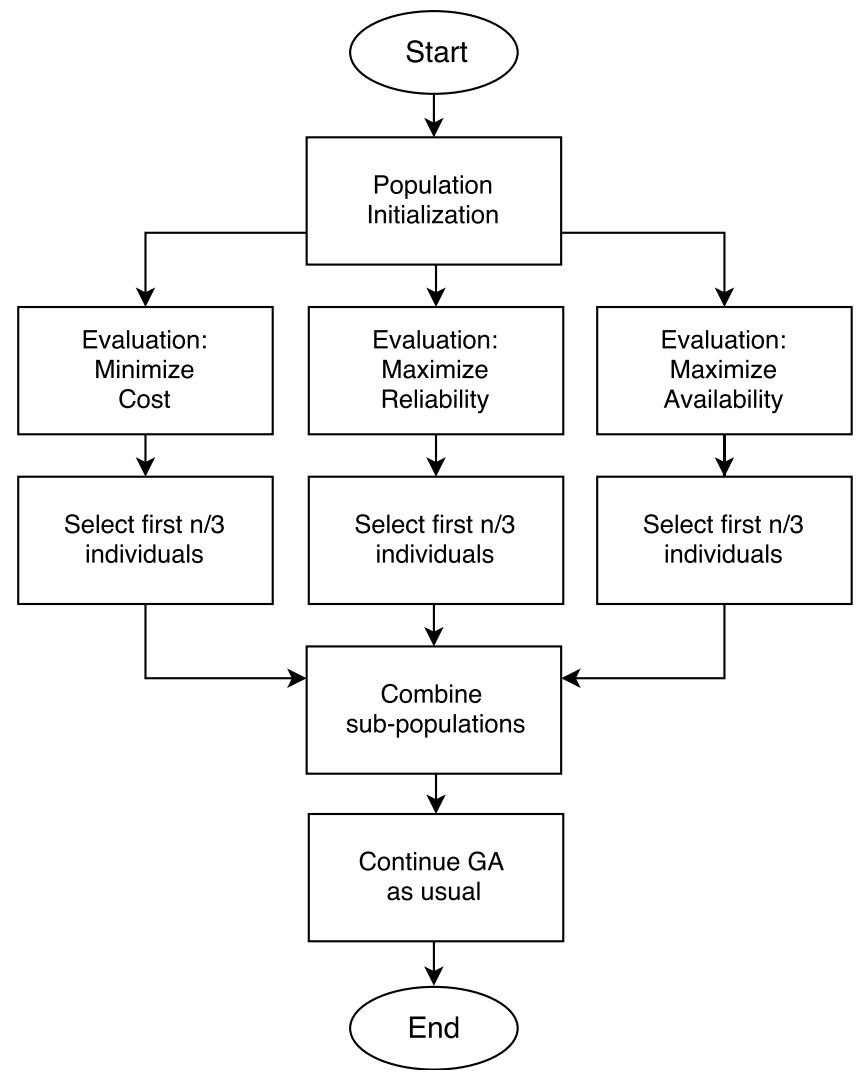

Figure 5. Flowchart of the approach inspired to the VEGA method, variant 1 .

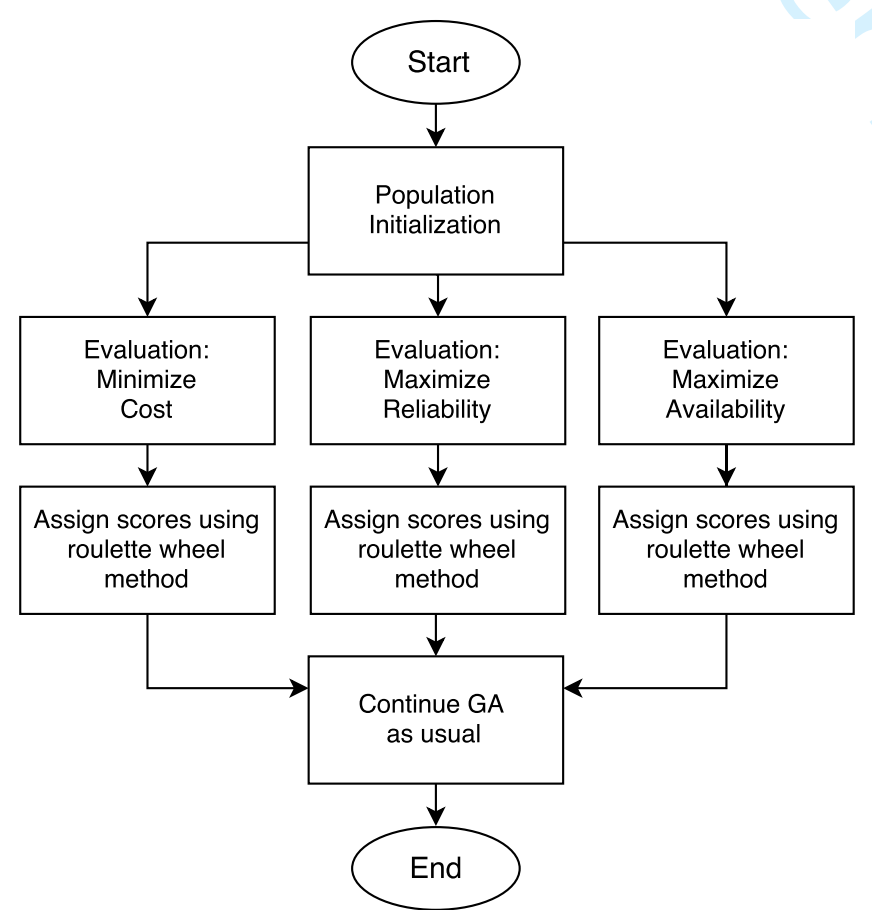

Figure 6. Flowchart of the approach inspired to the VEGA method, variant 2.

decision variables to the objectives is required. These should accurately characterize the links between the input parameters and the decision variables of the problem. The overall aim is to obtain the optimal value for each of the decision variables according to the preference of the decision-maker. The use of a characterization tool would be ideal to ensure that the evaluation of all the KPIs of the OWF is as accurate as possible, within the limits of the characterization model itself. However, this coupling is not a straightforward task, especially if the characterization tool is computationally expensive. As a consequence, the implementation of substitutive objective functions (a surrogate model) is required to evaluate each individual with respect to each objective. Hence, based on observations with the previously implemented characterization tool, specific objective functions are built. Furthermore, these objective functions are calibrated and benchmarked in order to ensure predictions are as close as possible to those that would be obtained with the characterization tool itself, previously validated independently and for which there is confidence in the model outputs (31).

When optimizing the O\&M assets of an offshore wind farm, a series of both strategic (long-term) and operational (on a daily basis) decision problems need to be addressed. In this work, only the strategic decisions, in terms of two main aspects, are considered: 1) the properties of the maintenance access systems and 2) the turbines components and their installed elements. Accordingly, the chromosome representing each individual in the GA includes decision variables for:

- the number of units for each access system potentially included in the maintenance fleet;

- whether each access system can perform maintenance interventions only during the day or also overnight;

- whether each kind of access system has been purchased or has to be chartered when it is required for maintenance;

- whether the availability of each kind of access system is limited to specific periods of the year (e.g. summer months) or not;

- whether for each component of the devices redundant elements should be installed (compatibly to technical constraints) or not;

- whether for each component of the turbines, a more reliable alternative should be installed (i.e. with a reduced failure rate) or not;

- whether for each component of the devices a repair or replacement intervention should be performable also overnight, in contrast to those components whose maintenance should be limited to periods with daylight; and

- whether for each component of the device there should always be an immediate availability of spare parts or not.

Under these circumstances, and based on the previous experiences with the O\&M characterization model, specific relationships are built in order to determine the fitness of each individual with respect to each of the three objective functions. These relationships constitute the surrogate model introduced earlier in the paper.

First, the contributions of the different parts of the chromosome to the cost objective function are determined by taking into accounts the following considerations:

- Number of units per access system: the more units available, the lower the risk of introducing delays in 
the maintenance of the farm, thus indirect reduction in cost due to reduced production losses. On the other hand, the expenses to hire or purchase an increased number of units will be higher. Therefore a balance is required in the ratio between the number of units and the number of maintenance interventions (both planned and unplanned). Besides, solutions in which two or more access systems are considered in a mixed maintenance fleet are favoured because the complementarity in their capabilities will be used to decrease lost production and repair costs (e.g. by using quicker and cheaper workboats for minor interventions and bigger vessels exclusively for medium or major interventions).

- Overnight operability of the access systems: if a vessel is able to perform maintenance interventions overnight there will be a reduction of the lost production as a consequence of a higher capacity of restoring the functionality of the device in case of failures. At the same time, an increase in direct O\&M cost has to be expected due to higher wages for the maintenance staff and other expenses (e.g. port fees) for the night shifts.

- Ownership of the access systems: if a set of vessels belongs to the farm the expenses due to mobilisations will be lower, the vessels will be more easily available for interventions (which reduces lost production) and and there will be no charter costs. On the other hand, the initial cost of purchase and the expenses related to port and maintenance fees of the vessels must be taken into account.

- Limited availability of the access systems: similarly to the previous points, a limited availability of the vessels, for instance during the summer months or when the vessels are chartered elsewhere, will increase the costs due to production losses; on the other hand, the port and maintenance fees, as well as staff costs and eventual hire expenses, will decrease.

- Redundancy of the components: the introduction of redundant elements in the components of the device will have a direct increase in cost due to the price of the redundant elements and their installation; on the other hand there will be an increase in the reliability of the device that, indirectly, will reduce the cost of interventions due to maintenance.

- Failure rate reduction of the components: analogously, better or more reliable components will be more expensive to purchase and replace in case of failure, but their replacement will be less frequent.

- Overnight operability of the components: likewise the overnight capability of the access systems, having components that can be maintained overnight reduces downtimes and production losses (indirect cost decrease). At the same time, additional expenses, then increase in costs, may be required to acquire components that can be repaired or replaced at any time.

- Immediate spare parts availability of the components: having spare parts for certain components always available means that eventual procurement times are null, reducing repair times and production losses. This comes at a cost, for instance due to higher investments in the spares and the need of bigger warehouse / engineering team to store / manage them.

Switching to the reliability objective function, this is dependent only on the taxonomy of the device and the configuration of its sub-assemblies. As such, the reliability of the devices is computed starting from the values of the components' failure rates, taking into account the placement of the components in series or in parallel systems, as well as eventual redundant items and the minimum number of these which are needed for the device to remain operational. Specific equations for such calculations can be found in (32). Furthermore, eventual overnight operability on the components indirectly increases their reliability through an increase in their repairability.

The third and last objective function is the availability. As this is usually measured as a percentage, where $0 \%$ corresponds to no energy production due to continuous downtime and $100 \%$ to the ideal production in the scenario of null downtime, the contributions to the objective function $A_{i}$ are calculated in such a way that in an ideal situation all the contributions would sum up to 100 , i.e. $\Sigma_{i} A_{i}<=100$. The values for each decision variable (or set of these) are then assigned proportionally to the relative importance of the contributions, whose ranges have been previously calibrated through comparison with the characterization tool (31). Hence, the same consideration made for the calculation of the cost objective functions on both the maintenance access systems and device components, can be used to calculate the scores in the availability objective function. The only difference is that in this case, i.e. for what concerns the availability only, there are no drawbacks (e.g. additional costs due to capital expenditures), so for example the higher the number of access systems the better, the more reliability related improvements the better, etc.

A qualitative summary of the impact of each decision variable on the three objective functions is provided in Table 2.

Table 2. Effect of each decision variables on the objective functions.

\begin{tabular}{cccc}
\hline Decision variable & Cost & Reliability & Availability \\
\hline Number of access system units & $\Uparrow, \downarrow$ & - & $\uparrow$ \\
Overnight operability of the access systems & $\Uparrow, \downarrow$ & - & $\uparrow$ \\
Ownership of the access systems & $\Uparrow, \downarrow$ & - & $\uparrow$ \\
Seasonality restrictions of the access systems & $\uparrow, \downarrow$ & - & $\downarrow$ \\
Redundancy measures on device components & $\Uparrow, \downarrow$ & $\Uparrow$ & $\Uparrow$ \\
Failure rate reduction of device components & $\Uparrow, \downarrow$ & $\Uparrow$ & $\Uparrow$ \\
Overnight repairability of device components & $\Uparrow, \downarrow$ & $\Uparrow$ & $\Uparrow$ \\
Immediate spares availability of device components & $\Uparrow, \downarrow$ & $\Uparrow$ & $\uparrow$ \\
\hline
\end{tabular}

Legend: $\Uparrow=$ Direct increase, $\Downarrow=$ Direct decrease, $\uparrow=$ Indirect increase, $\downarrow=$ Indirect decrease, - = No variation

Besides these considerations and correspondent contributions on the calculation of the respective objective functions, eventual penalties are used. Such penalties are introduced in order to consider some of the joint impacts of the decision variables that are not accurately captured by the implemented functions alone. It should be noted that though penalties are commonly used to handle constraints in an optimization problem, in this work these are used to ensure that the impact of interactions between decision variables are accurately captured in the objective functions. Consequently, a penalty in the calculation of the cost function is eventually introduced 
after considering the correlation between components maintenance categories and access systems maintenance categories (maintenance categories are used to classify the extent of an intervention, e.g. minor repair, major replacement, etc.). This reduces the probability of favouring possibly undesired solutions (e.g. with numerous units of expensive access systems that can be used only for a limited number of major repairs). Similarly, a further penalty in the cost function is possibly introduced to account for the indirect variations in cost due to variations in reliability. In other words, since a more reliable device requires less maintenance interventions and decreases the direct O\&M costs, solutions with a value of reliability far from the ideal situation with all the possible reliability related improvements in place (e.g. failure rate reductions and redundancy of the components) will be penalized.

Furthermore, constraints are applied at each step of the GA (any time that individuals are generated, crossed over and mutated) for the purpose of maintaining feasible solutions that either provide realistic solutions and satisfy the technical implications of the OWF management. These are:

- considering at least one unit of at least one access system in the maintenance fleet;

- not considering the properties of a vessel in the final solution if the vessel is not included in the fleet; and

- not assuming redundancy improvements if the preestablished possible number of redundant elements for a certain component is set to 0 due to technical requirements.

Finally, unlike in characterization models, the scores provided in the present optimization model are relative measures, to be treated as a unitless comparison metric to evaluate the relative quality of solutions rather than to interpret them as an absolute estimate of the performance indicator for that parameter. In fact, as the optimization procedure only compares solutions to one another, the true values with regards to the KPIs are not sought.

\section{Approach selection}

Given the formulation of the chromosome, its relationship to the O\&M strategy input parameters, and the objective functions, the three multi-objective GA approaches described are compared for a simplified case in order to provide an example of approach selection. Despite these are not generic rules, for the comparison in this specific problem the generated Pareto fronts are evaluated against these criteria: the more solutions in the area of the desired trade-offs (e.g. minimum cost / maximum reliability or maximum availability / maximum reliability) the better. This concept can be better visualized in Figure 7. In this figure, the Pareto fronts obtained for the three approaches are plotted considering only two objectives at a time in order to aid in clarity as exploring the Pareto surface in 3 dimensions is challenging as far more non-dominated solutions are needed to smoothly define the surface. The knee of the Pareto curves have been enlarged as this is often a key area of interest for a decisionmaker. This area includes those solutions that provide the most even trade-offs between the two objectives considered in each chart (e.g. maximum reliability at the minimum cost). It has to be remembered that though the presented plots look at projections onto two dimensions, all the solutions are considering all three objectives. Nonetheless, if for any reason one of the objectives is less relevant to the decisionmaker, the choice can be moved towards the other values on the Pareto (e.g. minimum cost regardless of reliability or availability values). It is important to note that though realistically a decision maker might generally be concerned with the knee, this is not always the case and in various situations the extents of the Pareto front also aid in the decision making process.

From the enlargements in these figures, the most effective approach in the search of the best trade-off solutions in this example is the one exploiting the weighted sum method. Despite very similar computational times, the solutions found with this method provide lower extremes in the cost/reliability and cost/availability Pareto fronts, as well as higher extremes in the availability/reliability Pareto front. Thus, although other approaches may provide more solutions on the Pareto front, preference is given to this method due to the presence of solutions lower in cost in the selected reliability and availability ranges. These quantitative decision criteria are illustrated in Table 3, where the minimum cost is in the selected reliability range, the minimum cost in the selected availability range, and the maximum availability in the selected reliability range are reported for figures $7 \mathrm{a}, 7 \mathrm{~b}$ and $7 \mathrm{c}$ respectively.

These are generic guidelines provided to explain how one might interpret and compare the results of different optimization approaches. Nonetheless, selection criteria will vary depending on the specific case analyzed and the preferences of the decision-maker.

Table 3. Values of solutions in the ranges selected according to the preferences of the authors for example in Figure 7.

\begin{tabular}{lrrr}
\hline Approach & S & W & V \\
\hline Minimum cost in reliability range 0.843 - 0.844 (Fig.7a) & 35546 & 14157 & 126096 \\
Minimum cost in availability range 64 - 68 (Fig.7b) & 40417 & 22209 & 71869 \\
Maximum availability in reliability range 0.835- - .845 (Fig.7c) & 69.06 & 69.81 & 58.45 \\
\hline
\end{tabular}

Legend: $\mathbf{S}=$ Superposition, $\mathbf{W}=$ Weighted Sum, $\mathbf{V}=$ VEGA inspired.

\section{Case study}

Based on the selection criteria above and the values in Table 4, the following section examines an example OWP optimized using the weighted sum approach. In fact, even though the superposition method may be used, because the economic metric for the selected reliability range is lower than in the weighted sum approach (first line of Table 4) and at least comparable within the selected availability range (second line of Table 4), due to the significantly higher availability metric in the selected reliability range (third line of Table 4) the weighted sum approach is preferred. In any case, despite only one approach is selected in order to simplify the application of the proposed methodology, nothing prevents the use of all three presented methods for a more effective comparison.

The case study considers Westermost Rough Wind Farm (33), a wind farm off the east coast of the United Kingdom which began operation in 2015. This wind farm consists of 35 Siemens SWT-6.0-154 turbines each rated at 6.0 MW. The O\&M port associated to this OWP is the 

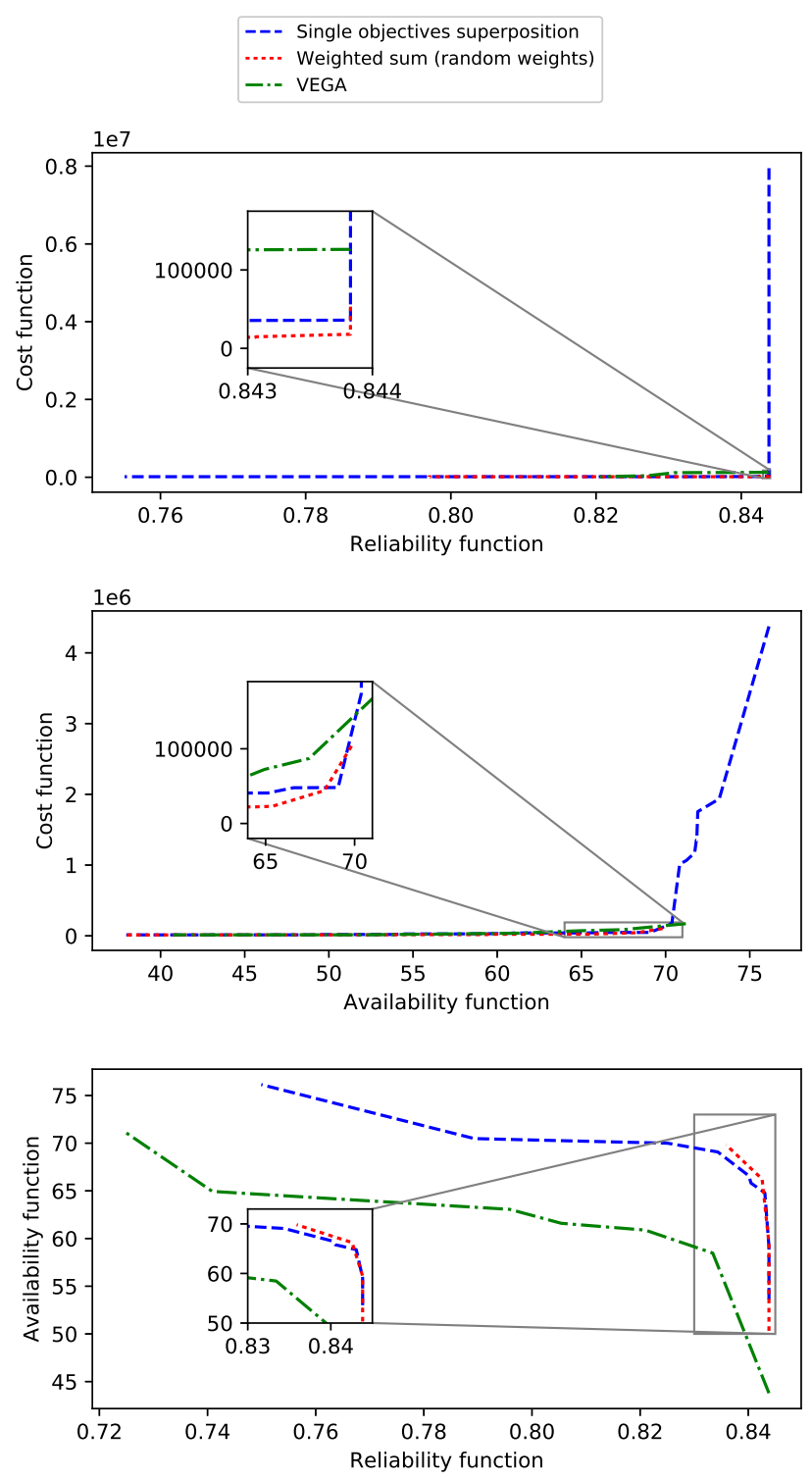

Figure 7. Comparison of the Pareto frontiers obtained using the three approaches.

Royal Dock Grimsby port (34), located approximately 40 $\mathrm{km}$ from the OWF, as shown in Figure 8. The MetOcean data needed for the calculation regarding energy produced and accessibility of the farm (wave height, wave period and wind speed) are retrieved using the numerical simulation model WAVEWATCH III (35) for the 10 year period from 1990 to 1999 with a timestep of 3 hours. The accessibility information, including weather windows and vessels transit times, are calculated for each day of the simulated lifetime using the offshore projects planning software Mermaid (Marine Economic Risk Management Aid) (36). A portbased O\&M strategy is assumed, in which the OWF can be maintained by means of three types of generic vessels capable of minor, medium and major maintenance interventions respectively. These are indicated in this work as crew transfer vessel (CTV), field support vessel (FSV) and heavy-lift vessel (HLV). The wave limits for the repair actions of these vessels, as well as the majority of the cost data, have been extracted from the literature (37; 38; 39). Where necessary, unknown economic values have been estimated based on industry experience and consistency with other economic values. Metocean limits and economic values associated to the access systems and used for this study are specified in Table 5. Even though only wave limits have been considered for the accessibility characterizations, wind and water current limits can be included if needed.

Table 4. Values of solutions in the ranges selected according to the preferences of the authors for the case study.

\begin{tabular}{lrrr}
\hline Approach & S & W & V \\
\hline Minimum cost in reliability range $0.0009815-0.0009816$ & $1.41 \mathrm{E} 6$ & $4.30 \mathrm{E} 6$ & $3.80 \mathrm{E} 6$ \\
Minimum cost in availability range 82.50-82.55 & $4.33 \mathrm{E} 9$ & $4.3 \mathrm{E} 9$ & $\mathrm{~N} / \mathrm{A}^{*}$ \\
Maximum availability in reliability range 0.00096-0.00098 & 69.70 & 82.42 & 67.10 \\
\hline Legend: $\mathbf{S}=$ Superposition, W = Weighted Sum, V = VEGA inspired. Note: ${ }^{*}=$ Not \\
present in selected range
\end{tabular}

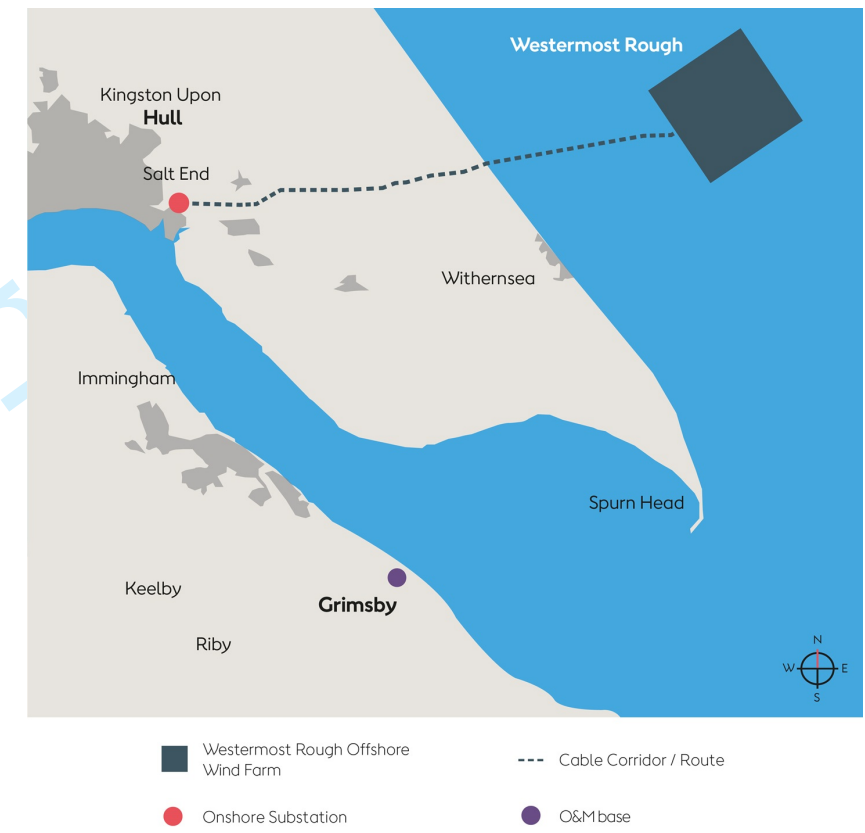

Figure 8. Map of Westermost Rough Wind Farm (33).

Table 5. Vessel cost parameters $(37 ; 38 ; 39)$.

\begin{tabular}{crrr}
\hline Access system & CTV & FSV & HLV \\
\hline Wave limit $\left(\mathrm{H}_{\mathrm{s}}, \mathrm{m}\right)$ & 1.5 & 1.5 & 2 \\
Transit speed $(\mathrm{knots})$ & 20 & 12 & 11 \\
\hline Day rate $(£)$ & 1750 & 9500 & 150000 \\
Standby day rate $(£)$ & 616 & 1232 & 2465 \\
Mobilization cost $(£)$ & 0 & 0 & 27000 \\
Transit fuel cost $(£)$ & 138 & 883 & 2187 \\
Average daily crew member cost $(£)$ & 220 & 220 & 220 \\
\hline
\end{tabular}

The components data for the devices, including failure rates and replacement costs, have been extracted from (40) by averaging over the values for the maintenance categories considered in the reference. Information on possible redundancies and other reliability related improvements have 
been assumed according to values reported in Table 6. An eventual failure rate reduction is assumed to halve the value of the original failure rate.

Table 6. Components parameters (40).

\begin{tabular}{lcccc}
\hline$\#$ & Component & Failure rate (f/yr) & Repair time $(\mathrm{hr})$ & Repair cost $(£)$ \\
\hline 1 & Pitch system & 1.076 & 89.0 & 65910 \\
2 & Generator & 0.999 & 67.0 & 25973 \\
3 & Blades & 0.520 & 31.2 & 18037 \\
4 & Lub system & 0.471 & 22.0 & 5253 \\
5 & Electrical comp. & 0.435 & 20.7 & 4550 \\
6 & Contactor & 0.430 & 17.5 & 4564 \\
7 & Controls & 0.428 & 17.5 & 4431 \\
8 & Safety & 0.392 & 13.2 & 4306 \\
9 & Sensors & 0.346 & 12.7 & 3995 \\
10 & Pumps, motors & 0.346 & 11.0 & 3544 \\
11 & Hub & 0.235 & 8.3 & 1126 \\
12 & Heaters/Coolers & 0.213 & 8.0 & 1075 \\
13 & Yaw system & 0.189 & 7.3 & 990 \\
14 & Tower/foundation & 0.185 & 7.0 & 918 \\
15 & Converter & 0.180 & 8.0 & 750 \\
16 & Transformer & 0.065 & 3.6 & 527 \\
\hline
\end{tabular}

Exploiting the values provided by the manufacturer for cut-in, cut-out and rated speed (41), the remaining intermediate values to acquire the complete power curve of the OWT are obtained by using the least square method. Despite the use of 6.0 MW wind turbines in the real project, in this case study the authors opted for the updated version of the turbine which is now rated at $8.0 \mathrm{MW}$, more representative of future OWPs. In fact, since two phases that include the current configuration with 35 OWTs and an extension of this with an additional 45 OWTs (for a total of 80 OWTs and $640 \mathrm{MW}$ ) are considered, the updated version of the OWT allows for a reduced number of devices to achieve the same total installed capacity. Finally, the strike price for the electricity generated by the OWF is assumed to be $£ 155 / \mathrm{MWh}$, the maximum possible for projects coming on line in 2015/2016 (42).

Once this set of input data is gathered, a first simulation is run with the characterization model in order to obtain a reference case, in which 3 CTVs, 1 FSV and 1 HLV (37) are assumed in the fleet, for each one of the two phases of the OWF. Consequently, a new simulation with the input data provided by the execution of the GA is repeated. In this way, the values on the KPIs of the OWF can be compared before and after the optimization and the differences quantified.

\section{Phase 1 - 35 OWTs}

By running the GA using the weighted sum approach and the inputs described in the previous section, the Pareto frontiers illustrated in Figure 9 are obtained. From these, one solution (indicated by the data cursor) is selected among those obtained in the cost / availability chart, and decoded in terms of the corresponding O\&M strategy shown in Table 7. The O\&M strategy indicated by the optimization algorithm in Table 7 is then re-evaluated using the characterization tool, yielding the values presented in Table 8. As shown in Table 8, the selection of the optimized maintenance strategy allows for significant reductions of lost production by $74 \%$ and O\&M costs by $33 \%$. This, in turn, generates an increase in energy production and availability by $3 \%$, with this last passing from $95.64 \%$ to $98.88 \%$, and an increase of the generated income by almost $5 \%$, which translates in additional m£ 75 over the 10 years of the considered lifetime.

Table 7. Input variables according to decoded solution for phase 1.

\begin{tabular}{lc}
\hline Objective functions values & \\
\hline Cost function & $10.23 \times 10^{6}$ \\
Reliability function & $9.81 \times 10^{-4}$ \\
Availability function & 67.33 \\
\hline Access systems decision variables & \\
\hline Use combinations of access systems: & Yes \\
Number of units available: & No (CTV), 7 (FSV), 5 (HLV) \\
Vessel(s) purchase: & Yes (HLV) \\
Overnight operability: & No (All vessels) \\
Seasonality restrictions: & $4,5,6,9,12,13$ \\
\hline Device related decision variables & $1,2,4,5,6,7,9,10,11,12,13,14$ \\
\hline Redundancy measures on components: & $1,2,5,8,10,12,13,16$ \\
Failure rate reduction on components: & $3,4,6,7,10,11,15$ \\
Overnight operability on components: & \\
Spares immediate availability for components: &
\end{tabular}

The O\&M strategy indicated by the optimization algorithm in Table 7 is then re-evaluated using the characterization tool, yielding the values presented in Table 8.

Table 8. Comparison of the results obtained for phase 1 before and after the optimization indicated by the GA.

\begin{tabular}{lrrr}
\hline Quantity & Value $1^{\star}$ & Value $2^{\star \star}$ & Variation (\%) \\
\hline Average annual energy (GWh) & 1057.38 & 1093.68 & 3.38 \\
Average annual loss (GWh) & 48.21 & 12.43 & -74.22 \\
Capacity factor (\%) & 43.09 & 44.54 & 3.37 \\
Equivalent hours & 3776.39 & 3904.17 & 3.38 \\
Availability (\%) & 95.64 & 98.88 & 3.39 \\
Total gross production over 10 years (m£) & 1638.95 & 1694.41 & 3.38 \\
Total lost production over 10 years (m£) & 74.72 & 19.26 & -74.22 \\
Total O\&M costs - Repairs, vessels and crew (m£) & 58.49 & 38.93 & -33.44 \\
Total generated income over 10 years (m£) & 1580.45 & 1655.49 & 4.75 \\
\hline
\end{tabular}

Note: ${ }^{*}=$ Before optimization, ${ }^{* *}=$ After optimization

\section{Phase 2 - 80 OWTs}

The evaluation of the KPIs and the optimization using GAs are repeated for the second phase of the case study. The Pareto frontiers obtained for this phase are shown in Figure 10, with the solution of interest highlighted in the same figure, corresponding O\&M strategy parameters shown in Table 9, and the comparison of the KPIs shown in Table 10.

Table 9. Input variables according to decoded solution for phase 2.

\begin{tabular}{lc}
\hline Objective functions values & \\
\hline Cost function & $12.84 \times 10^{6}$ \\
Reliability function & $9.81 \times 10^{-4}$ \\
Availability function & 69.48 \\
\hline Access systems decision variables & \\
\hline Use combinations of access systems: & Yes \\
Number of units available: & No \\
Vessel(s) purchase: & Yes (FSV) \\
Overnight operability: & No (All vessels) \\
Seasonality restrictions: & $4,5,6,9,12,13$ \\
\hline Device related decision variables & $1,4,5,6,9,12,13,16$ \\
\hline Redundancy measures on components: & $2,3,8,10,11,12,13,16$ \\
Failure rate reduction on components: & $2,3,4,5,6,7,8,9,10,12,16$ \\
Overnight operability on components: & \\
Spares immediate availability for components: &
\end{tabular}



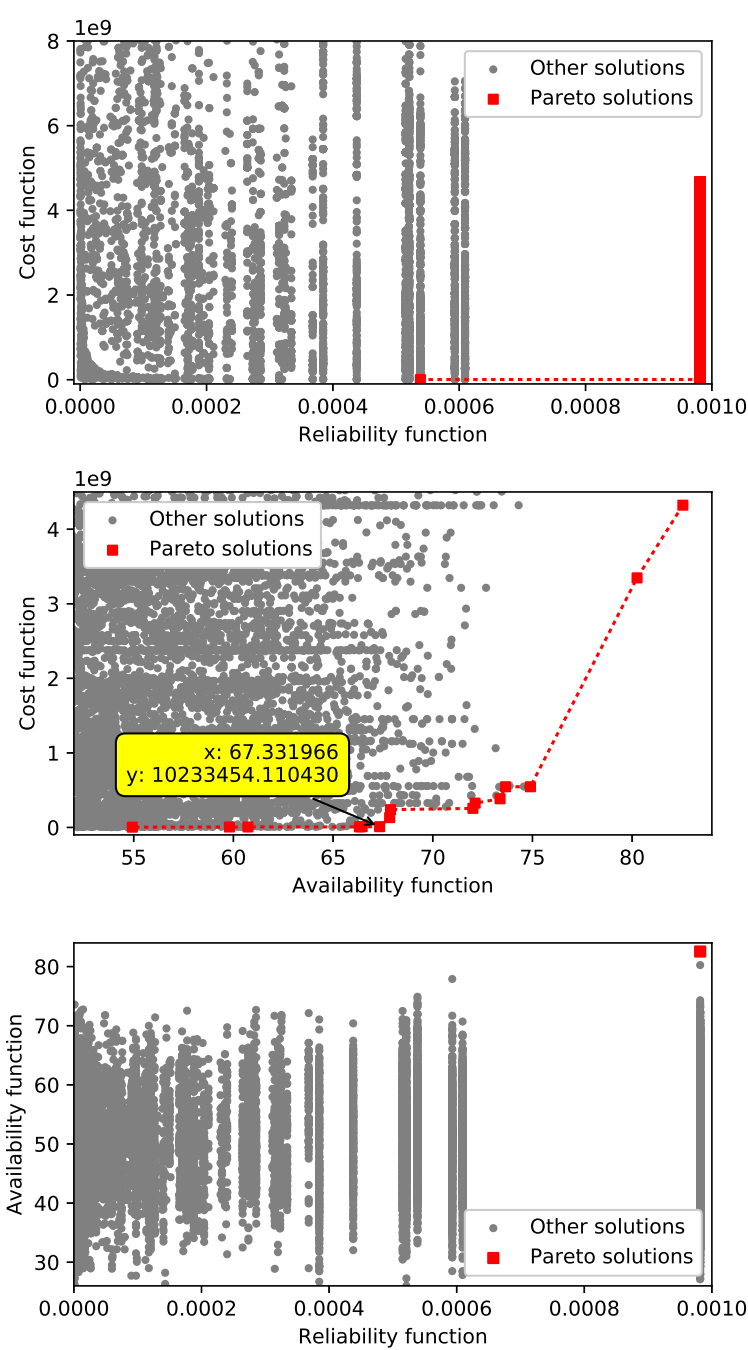

Figure 9. Pareto frontiers obtained for phase 1 using the weighted sum approach. Solutions plotted in terms of their cost and reliability values in Figure 9a, cost and availability values in Figure $9 \mathrm{~b}$ and availability and reliability values in Figure $9 \mathrm{c}$

Table 10. Comparison of the results obtained for phase 2 before and after the optimization indicated by the GA.

\begin{tabular}{|c|c|c|c|}
\hline Quantity & Value $1^{*}$ & Value $2^{\star \star}$ & Variation (\%) \\
\hline Average annual energy (GWh) & 1757.11 & 2497.04 & 42.11 \\
\hline Average annual loss (GWh) & 769.97 & 30.03 & -96.10 \\
\hline Capacity factor $(\%)$ & 31.32 & 44.51 & 42.11 \\
\hline Equivalent hours & 2745.48 & 3901.63 & 42.11 \\
\hline Availability (\%) & 69.53 & 98.81 & 42.11 \\
\hline Total gross production over 10 years $(\mathrm{m} £)$ & 2723.52 & 3870.42 & 42.11 \\
\hline Total lost production over 10 years $(\mathrm{mE})$ & 1193.45 & 46.558 & -96.10 \\
\hline Total O\&M costs - Repairs, vessels and crew (m£) & 102.21 & 103.99 & 1.74 \\
\hline Total generated income over 10 years $(\mathrm{mE})$ & 2621.30 & 3766.43 & 43.69 \\
\hline
\end{tabular}

Similar to the previous situation, also for the second simulated phase the optimized O\&M strategy allows for the achievement of reduced losses and increased production, as shown in Table 10. In this case, the relative variations are more significant than in the first phase, with the availability increasing from around $70 \%$ to more than $98 \%$ and the capacity factor rising from $31 \%$ to $44 \%$. As a consequence, the total lost production lowers to m£46 over the 10 years lifetime, and the generated income increases by almost $44 \%$, meaning an average additional gain of $\mathrm{m} £ 114$ per year.
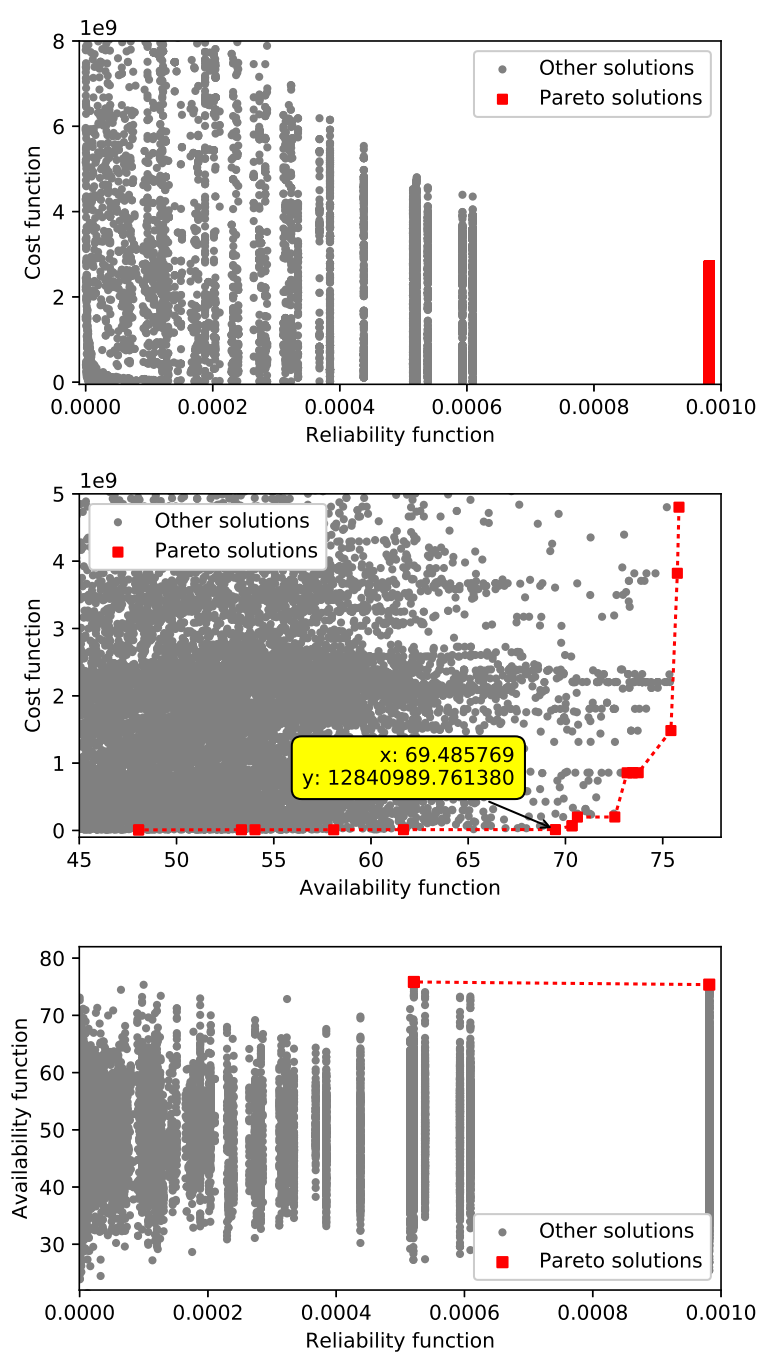

Figure 10. Pareto frontiers obtained for Phase 2 using the weighted sum approach. Solutions plotted in terms of their cost and reliability values in Figure $10 \mathrm{a}$, cost and availability values in Figure $10 \mathrm{~b}$ and availability and reliability values in Figure 10c.

However, the O\&M cost slightly increase (by less than 2\%) compared to the base case scenario.

\section{Discussion}

The weighted sum method is used to illustrate the results obtainable with the proposed methodology. However, the selection of the most appropriate approach is generally casespecific, and in some cases even a combination of them might be beneficial in order to obtain better solutions according to the priorities of the decision-maker. Furthermore, a more thorough comparison of the methodologies should compare their computational complexity as each of the methods does not intrinsically require the same number of evaluations. Although the parameters of the GA (number of generations, number of individuals, etc.) do not vary, the final solutions are obtained by letting random individuals evolve according to different evolutionary paths in each approach and then analyzing the aggregated results. If necessary, crossover and mutation rates can be left as variable and able to self-adapt depending on pre-established rules and feedback from each optimisation cycle. Similarly, the number of generations can 
be left as a function of the relative improvement between two consecutive Pareto fronts, and a maximum computational time or number of generations used as stopping criterion.

In this work, the conditions for each path are dictated by the objective functions in the first approach proposed, the values of the weights at each iteration in the second and the selection criteria in the third. As a result, the number of proposed individuals, and therefore the number of evaluation calls required for each of the three approaches may be unequal if the parameters are not properly tuned. The three approaches may therefore, be unfairly compared.

From the execution of the GAs, a series of candidate solutions, representing a set of input data for the characterization tool, are obtained. Due to the difficulty in interpreting the results and choosing useful candidates when these solutions are plotted in terms of the three objective functions considered (cost, reliability and availability) simultaneously (e.g. in a 3D scatter diagram), the charts providing a visual representation of the solutions (and associated Pareto fronts) in terms of two objective functions at a time are preferred. In the present work, the authors opted to prioritize the profitability of the project. As a consequence, decisions are principally based on the cost / availability chart because these two criteria are more relevant from a profitability perspective. This is a preference of the authors in order to illustrate the case study, but a decision-maker could use other criteria according to his/her priorities. Nevertheless, the associated value for the third objective function, the reliability, can be used in order to refine the choice between two or more neighbour solutions in the Pareto frontiers.

In the charts where the reliability is considered, a discretization of the solutions can be observed due to the constraints which allow only certain combinations of redundancy and failure rate reductions for the components of the device. The cost / availability charts, however, appear continuous in the objective space resulting in a more well defined Pareto front. As the two phases considered in this case study differ only in the number of turbines, with all other parameters held constant, a similar distribution of solutions can be observed in the presented Pareto fronts. The criteria used for the selection of the optimized solution in both charts looked at obtaining an as high as possible value of availability while keeping the value of the cost function as low as possible. Therefore the solutions are selected from the lower right portion of the charts, prior to the cost function rising steeply. This prioritizes the cost variation eliminating excessively high cost solutions (especially after taking into account the large variations of the cost function on the $y$ axis).

Even though the resulting distribution of candidate solutions (and as a consequence the choice of the optimized solution) is similar for both the phases of the OWF, the selected optimal O\&M strategies to be tested with the characterization tool differ due to the increased number of turbines in Phase 2. As a result of the increase in the turbines of the OWF, the number of maintenance interventions increases and the costs dynamic changes. As a consequence, a larger fleet is required and it is necessary to select a number of access systems more appropriate for the number of repairable components which can be repaired by that type of vessel. Similarly, while the full availability without seasonal restrictions is required in both cases, the overnight operability is necessary only for major interventions with the HLV in the first phase, whereas for the second phase it is needed also for minor operations with the CTVs.

Turning to the component specific decision variables, thanks to the higher number of vessels available less components with lower failure rate and repairable or replaceable overnight are required to achieve the desired values in the second case. On the other hand, due to the increased number of devices, a greater number of components require the immediate availability of spare parts when compared to phase 1. In addition, in order to cope with the higher number of repairs, more maintenance interventions are needed. This increases the direct O\&M costs, causing also a raise in the optimized strategy with respect to the base case scenario due to the higher number of vessels required, but which is still highly compensated by the reduced downtime production.

Of course, these choices and improvements on the devices have a cost due to the installation of more expensive components, redundant elements and spare parts, but also to higher vessel charters, crew compensations and port expenses. These additional expenses are calculated using the same formulation of the optimization framework, and their value estimated around the $13 \%$ and $8 \%$ of the final O\&M costs respectively for the 2 simulated phases. Even if these values are rough approximations, the additional expenses are highly compensated by the significant increases in energy production and final generated income, as shown in Tables 8 and 10. The introduced improvements result in a significant reduction of the energy losses due to downtime and of the O\&M costs due to repairs and replacements and use of vessel. This, in turn, increases the availability and profitability of the OWP for both the simulated phases, reaching considerably high value for typical OWFs. Firstly, these variation depend on the reference case, based on the literature, selected for this work, and could vary accordingly if other options are considered. Secondly, this gain is also due to the relatively high Contract for Difference (CfD) assumed for this project. Lower CfDs would result in less effective improvements, as well as potentially generate very different strategies during the search for optimized solutions and orientate the selection according to different criteria (e.g. lower costs rather than higher availability). However, reasonable improvements can be expected also with lower CfDs or other compensation schemes. For instance, with a CfD price of $£ 57.50 / \mathrm{MWh}$ according to the last CfD auction for OWPs scheduled for commissioning in 2022/23 (43), the generated income over the 10 years of simulated lifetime would lower to m£589 and m£1331 for the first and second phase respectively, leaving a reasonable margin of profit even after deducting the additional expenses due to improvements.

This effect is more observable for the second phase of the wind farm where, although the optimization process results in a marginal availability increase, the impact on production and revenue is significant. In the first phase of the wind farm, however, a more significant impact on the availability as a result of the optimization process is observed. This is in part due to the modest results obtained before the optimization, when the base input set is less suitable because of the high 
number of wind turbines. This reflects the major difficulty in managing the maintenance assets when the number of devices to operate and maintain increases, but also shows the increased importance of using optimization models for larger OWFs.

Finally, considering the maintenance assets optimization within a mainly corrective framework, with a restricted perspective on the scheduled and predictive maintenance, limits the use of the surrogate model as a complete alternative to O\&M characterization tools.

\section{Conclusions}

In this paper, a novel approach utilizing genetic algorithms is used for the optimization of the maintenance assets of an offshore wind farm. In this approach, specific simplified relationships are built and implemented in order to evaluate the quality of each generated solution based on previous experiences with a computational tool for the accurate estimation of the key performance indicators of an offshore renewable project. Three methods are proposed for the automated evolution of the candidate solutions towards the most desirable combinations of decision variables according to multiple objectives. One of these, the weighted sum approach, is selected and a case study deploying it is presented together with the benefits of using such method.

Though the methodology aims to automate part of the decision making process, the final decisions from the Pareto front still require the interpretation of the optimization results and some engineering judgment. This is especially true when discerning between similar solutions. The main challenge in the present methodology is adequately establishing the simplified mathematical relationships that link the parameters of the project to the decision variables on which there is uncertainty. Nevertheless, similar to the development of other models, confidence on the evaluation functions is acquired and, eventually, the relationships refined by testing the model for a series of different cases and comparing the outputs with expectations or results provided by other methods in an iterative refinement process. Comparisons and feedback on the optimization framework allow for the reinforcement or confutation of the foundations used to build it (e.g. a previously implemented characterization tool). For instance, following the optimization procedure, all the improvements have to be compared against the direct costs of their implementation in order to also consider the drawbacks of a solution. As a consequence, if a separate characterization model is used to verify the effectiveness of the modifications (like in the case of this research) this will have to be modified in such a way to be capable to measure those additional capital or development expenses.

Under these circumstances, this work constitutes one of the two parts of a future wider computational framework that includes characterization and optimization of the O\&M assets of an offshore renewable project within the same tool, seeking the automated optimization of the O\&M assets according to an accurate estimations of the project's KPIs.

Further work shall extend the validity of the demonstrated approach by using more complete and accurate data. A reliable optimization requires in fact an extensive knowledge of all the economic and technical characteristics of the offshore project. Similarly, other approaches based on both nature-inspired and not optimization methods can be tested for more cases in order to assess the best methodology for each case or type of problem. This, in turn could be further extended by finding the rules or indications to systematically tune the parameters that regulate the algorithm.

\section{Acknowledgements}

The first author was funded by the Marie Curie Actions of the European Union's Seventh Framework Programme FP7/20072013/ under REA grant agreement number 607656 (OceaNet project) and by the industrial partner James Fisher Marine Services Ltd. Mojo Maritime (JFMS group) have provided access to Mermaid to support, and for integration with, this research. This work is also funded by the EPSRC (UK) grant for the SuperGen United Kingdom Centre for Marine Energy Research (UKCMER) [grant number: EP/P008682/1]

\section{References}

[1] Rodrigues S, Restrepo C, Kontos E et al. Trends of offshore wind projects. Renewable and Sustainable Energy Reviews 2015; 49: 1114-1135. DOI:10.1016/j.rser.2015.04.092.

[2] Poulsen T, Hasager CB and Jensen CM. The role of logistics in practical levelized cost of energy reduction implementation and government sponsored cost reduction studies: Day and night in offshorewind operations and maintenance logistics. Energies 2017; 10(4). DOI:10.3390/en10040464.

[3] Dalgic Y, Lazakis I and Turan O. Investigation of Optimum Crew Transfer Vessel Fleet for Offshore Wind Farm. Wind Engineering 2015; 39(1): 31-52.

[4] Stålhane M, Vefsnmo H, Halvorsen-Weare EE et al. Vessel Fleet Optimization for Maintenance Operations at Offshore Wind Farms under Uncertainty. Energy Procedia 2016; 94(1876): 357-366. DOI:10.1016/j.egypro.2016.09.195.

[5] Marseguerra M, Zio E and Podofillini L. Multiobjective spare part allocation by means of genetic algorithms and Monte Carlo simulation. Reliability Engineering and System Safety 2005; 87(3): 325-335. DOI:10.1016/j.ress.2004.06.002.

[6] Igba J, Alemzadeh K, Henningsen K et al. Effect of preventive maintenance intervals on reliability and maintenance costs of wind turbine gearboxes. Wind Energy 2015; 18: 2013-2024. DOI:10.1002/we.1801.

[7] Javanmard $\mathrm{H}$ and Koraeizadeh AaW. Optimizing the preventive maintenance scheduling by genetic algorithm based on cost and reliability in National Iranian Drilling Company. Journal of Industrial Engineering International 2016; 12(4): 509-516. DOI:10.1007/s40092-016-0155-9.

[8] Li X, Ouelhadj D, Song X et al. A decision support system for strategic maintenance planning in offshore wind farms. Renewable Energy 2016; 99: 784-799. DOI:10.1016/j.renene. 2016.07.037.

[9] Hofmann M and Sperstad IB. NOWIcob - A tool for reducing the maintenance costs of offshore wind farms. Deepwind'2013 - Selected Papers From 10Th Deep Sea Offshore Wind R\&D Conference 2013; 35(January): 177-186. DOI:10.1016/j.egypro.2013.07.171.

[10] Besnard F, Fischer K and Tjernberg LB. A model for the optimization of the maintenance support organization for offshore wind farms. IEEE Transactions on Sustainable 
Energy 2013; 4(2): 443-450. DOI:10.1109/TSTE.2012. 2225454 .

[11] Dalgic Y, Lazakis I, Dinwoodie I et al. Advanced logistics planning for offshore wind farm operation and maintenance activities. Ocean Engineering 2015; 101: 211-226. DOI: 10.1016/j.oceaneng.2015.04.040.

[12] Hofmann M. A Review of Decision Support Models for Offshore Wind Farms with an Emphasis on Operation and Maintenance Strategies. Wind Engineering 2011; 35(1): 116. DOI:10.1260/0309-524X.35.1.1.

[13] Shafiee M and Sørensen JD. Maintenance optimization and inspection planning of wind energy assets: Models, methods and strategies. Reliability Engineering and System Safety 2017; In press: 1-19. DOI:10.1016/j.ress.2017.10.025.

[14] Rinaldi G, Thies PR, Walker R et al. On the Analysis of a Wave Energy Farm with Focus on Maintenance Operations. Journal of marine science and engineering 2016; 4(3). DOI: 10.3390/jmse4030051.

[15] Rinaldi G, Thies P, Walker R et al. A decision support model to optimise the operation and maintenance strategies of an offshore renewable energy farm. Ocean Engineering 2017; 145: 250-262. DOI:10.1016/j.oceaneng.2017.08.019.

[16] Alexander D. Application of Monte Carlo simulation to system reliability analysis. In 20th international pump users symposium. pp. 91-94.

[17] Rinaldi G, Thies PR and Johanning L. A coupled Monte Carlo - Evolutionary Algorithm approach to optimise offshore renewables O \& M. In 12th European Wave and Tidal Energy Conference. Cork, pp. 1-7.

[18] Wang L, Kolios A, Nishino T et al. Structural optimisation of vertical-axis wind turbine composite blades based on finite element analysis and genetic algorithm. Composite Structures 2016; 153(December): 123-138. DOI:10.1016/j.compstruct. 2016.06.003.

[19] Gonzalez-Longatt FM, Wall P, Regulski P et al. Optimal electric network design for a large offshore wind farm based on a modified genetic algorithm approach. IEEE Systems Journal 2012; 6(1): 164-172. DOI:10.1109/JSYST.2011. 2163027.

[20] Dahmani O, Bourguet S, Machmoum M et al. Optimization and reliability evaluation of an offshore wind farm architecture. IEEE Transactions on Sustainable Energy 2017; 8(2): 542-550. DOI:10.1109/TSTE.2016.2609283.

[21] Pillai A, Chick J, Johanning L et al. Optimisation of Offshore Wind Farms Using a Genetic Algorithm. International Journal of Offshore and Polar Engineering 2016; 26(3): 225234. DOI:10.17736/ijope.2016.mmr16.

[22] Holland J. Adaptation in natural and artificial systems. Ann Arbor: University of Michigan Press, 1975.

[23] Man KF, Tang KS and Kwong S. Genetic Algorithms: Concepts and Applications. IEEE Transactions on industrial electronics 1996; 43(5): 519-534. DOI:10.1109/41.538609.

[24] Aggarwal, Shaifali, Richa Garg PG. A Review Paper on Different Encoding Schemes used in Genetic Algorithms. Advanced Research in Computer Science and Software Engineering 2014; 4(1): 596-600. URL http://www.ijarcsse.com/docs/papers/ Volume \{_\} 4/1\{_\} January2014/V4I1-0373.pdf.

[25] Konak A, Coit DW and Smith AE. Multi-objective optimization using genetic algorithms : A tutorial. Reliability
Engineering and System Safety 2006; 91: 992-1007. DOI: 10.1016/j.ress.2005.11.018

[26] Zitzler E and Thiele L. Multiobjective evolutionary algorithms: a comparative case study and the strength Pareto approach. IEEE Transactions on Evolutionary Computation 1999; 3(4): 257-271. DOI:10.1109/4235.797969.

[27] Marler RT and Arora JS. The weighted sum method for multi-objective optimization: New insights. Structural and Multidisciplinary Optimization 2010; 41(6): 853-862. DOI: 10.1007/s00158-009-0460-7.

[28] Schaffer J. Multiple objective optimization with vector evaluated genetic algorithms. The 1st international Conference on Genetic Algorithms 1985; : 93-100URL http://dl.acm.org/citation. cfm?id=657079.

[29] Deb K. Multi-objective optimization using evolutionary algorithms. Xix ed. Chichester, London: Wiley, 2001.

[30] Grefenstette J. Optimization of Control Parameters for Genetic Algorithms. IEEE Trans Systems, Man, and Cybernetics 1986; SMC-16(1): 122-128. DOI:10.1109/ TSMC.1986.289288.

[31] Rinaldi G, Pillai AC, Thies PR et al. Verification and benchmarking methodology for O\&M planning and optimization tools in the offshore renewable energy sector (submitted). In International Conference on Ocean, Offshore and Arctic Engineering (OMAE). Madrid, 2018.

[32] Rome Laboratory. Reliability Engineer's Toolkit. April, New York, 1993.

[33] Westermost Rough Wind Farm, 2017. URL https: //orsted.co.uk/en/Generatingenergy/ offshorewind/Ourwindfarms. Last accessed: 12 December 2017.

[34] Royal Dock Grimsby. URL http://www. portofgrimsby.com/index.html. Last accessed: 12 December 2017.

[35] Tolman HL, Balasubramaniyan B, Burroughs LD et al. Development and Implementation of Wind-Generated Ocean Surface Wave Models at NCEP. American Metereological Society 2002; : 311-333DOI:10.1175/1520-0434(2002) 017<0311:DAIOWG>2.0.CO;2.

[36] Morandeau M, Walker RT, Argall R et al. Optimisation of marine energy installation operations. International Journal of Marine Energy 2013; 3-4: 14-26. DOI:10.1016/j.ijome. 2013.11.002.

[37] Dinwoodie I, Endrerud OeV, Hofmann M et al. Reference Cases for Verification of Operation and Maintenance Simulation Models for Offshore Wind Farms. Wind Engineering 2015; 39(1): 1-14. DOI:10.1260/0309-524X.39. 1.1 .

[38] Tavner PJ. Offshore wind turbines : Reliability, availability and maintenance. Digital Library: Institution of Engineering and Technology, 2012. ISBN 978-1-84919-229-3.

[39] Katsouris G and Savenije LB. Offshore Wind Access 2017. Technical report, ECN, 2017.

[40] Carroll J, Mcdonald A and Mcmillan D. Failure rate, repair time and unscheduled O\&M cost analysis of offshore wind turbines. Wind Energy 2015; 19(6): 1107-1119. DOI:10. 1002/we.

[41] Siemens wind turbine data. URL http: //www . energy . siemens.com/hq/en/renewable-energy/ wind-power. Last accessed: 12 December 2017. 
[42] Department of Energy \& Climate Change. Investing in renewable technologies - CfD contract terms and strike prices. Technical Report December, 2013. URL https:

//www.gov.uk/government/publications/

investing-in-renewable-technologies-cfd-contract-terms-and-strike-prices.

[43] Contracts for Difference news. URL https:

//www.offshorewind.biz/2017/09/11/

three-offshore-wind-projects-secure-contracts-for-difference-as-strike-prices-go-down/.

Last accessed: 12 December 2017. 